\title{
Cloud Computing Technology Research Oriented Smart Grid
}

\author{
Wang XiaoKan ${ }^{1, a}$, Chen Dongzhao ${ }^{1, b}$ \\ ${ }^{1}$ Henan Mechanical and Electrical Vocational College, Zhengzhou,Henan,China \\ awangxiaokan@126.com, bwxkbbg@163.com
}

Keywords: cloud computing;smart cloud; smart grid; distributed; resource management; safety strategy

Abstract. With the continuous development of smart grid scale, smart grid would be realized in the power system and it has increasingly demands for processor resources and storage resources of the power system. Proposed smart cloud of power system which could make use of the existing intelligent processors and storage devices to the current resources by using the internal network of power system.All that will improve the calculation and storage capacity and data security of the overall system, and reduce the investment of system expansion, and provide ideas and strong technical support for smart grid and super scale computing.

\section{Introduction}

At present, the world's energy situation is undergoing a profound transformation. In order to improve network efficiency, reduce operating costs and maximize the value of the grid,it needs to promote the development of low-carbon, high efficient and clean electric power industry,restructure grid information loop and build new user feedback model.So all the countries in the world have turned their attention to the smart grid technology field and countries around the world will turn their attention to the smart grid technology and launched a sweeping global smart grid construction boom.

However, with the smart grid technology unceasing development, the future data and information of electric power system will be explosive growth, it is no doubt that it brings enormous challenge for the system operation and advanced analysis. The existing hardware infrastructure and computing power of power system was very difficult to adapt to the computing power and storage requirements of the future power system on-line analysis and real-time control.Therefore, in the case of maintaining existing hardware facilities unchange,the new computing technology was urgent to introduce that it was good for the full integration of computing power and memory resourcesin the internal grid system,enhancing smart grid ability of on-line analysis and real-time control and providing effective technical support for smart grid.

Cloud computing is a new computing model, it will provide a set of distributed computer system related ability for the users or applications with transparent services and allows access to needed services through the network under the understanding service technology, no-related knowledge and operation ability.

Because cloud computing has the characteristics of distributed computing,storage properties, easy expansion and management, it is especially suitable to solve a series of new problems which brings from the smart grid technology.Therefore, in order to improve the real-time control and advanced analysis ability,provide effective support for the smart grid technology development and integrate data resources and processor resources of on the current system, cloud computing is introduced into the smart grid technology that the existing power system hardware and infrastructure basically are unchanged,i it has no doubt that it has an extremely important research value and significance.

\section{Cloud Computing Technology}

Cloud computing technology is still in the development stage, the global research institutions, IT company and industry experts have not yet formed a unified opinion of the calculation including the main technical characteristics,key features and specific functions,so the cloud computing technology has not formed the unified and clear definition.Generally, cloud computing is a distributed processing, 
parallel processing and the development of grid computing, or the commercial implementations of computer science concepts which is based on the network computing mode.

The core of cloud computing is virtualization. Virtualization allows cloud computing system to provide dynamic computing resources according to the good service rank agreement of service provider and customer. In essence, cloud computing could store the data, applications and services in the cloud, and could make full use of the powerful computing ability of a group of interconnection virtual machine which composed of parallel and distributed computing system to realize the adaptability of user business system.

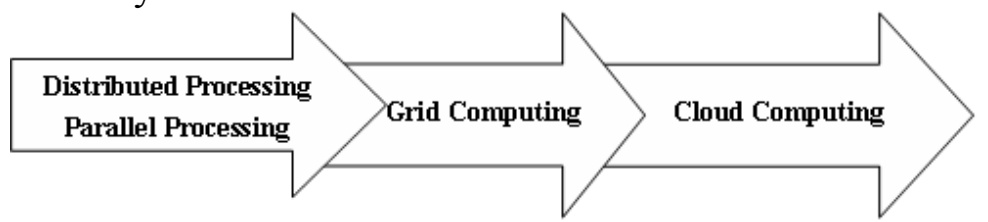

Fig. 1 Development Process of Cloud Computing

Cloud computing is a new type shared computing model based of infrastructure and network, it could aggregate resources gathering and sharing, resources, open and unified access, resource simulation and isolation which allows users or applications in a transparent way to use all kinds of resources without paying attention to all kinds of resources in detail.It has the ability of users dynamic adaptive service, so that each user feels themselves use the resources.

Although cloud computing technology is developing rapidly and has become the development trend of the next generation of IT, it still has insufficient of the solution of data security and privacy, system ability, availability, reliability, manageability and cloud interaction problems and needs further exploration and research.All manuscripts must be in English, also the table and figure texts, otherwise we cannot publish your paper.

\section{Cloud Computing Oriented Smart Grid}

\section{The Concept of Intelligent Cloud}

With development of smart grid technology and the formation of national interconnected power grid, the data and information of the future power system will become more and more complex which is growing with geometrical progression, and all kinds of information correlation the will also be more and more closely. Meanwhile, the power system online dynamic analysis and the required computing power will be dramatically improved, the computing power of the current power system has been difficult to adapt to the requirement of new applications. The growing amount of data put forward the new requirement to the data processing capacity of grid company 's information system. In this case, power companies have been impossible using the traditional mode of investment which relying on replacing a large number of computing devices and storage devices to solve the problem, so the new technologies must be applied in the grid system to fully exploit the existing potential of the power system hardware and to improve its applicability and utilization.

Cloud computing could be introduced into the power system based on the above concept to construe cloud computing system oriented smart grid and to form the private cloud of power system-smart cloud. Smart cloud takes full advantage of the power system's own physical network, and integrates of existing computing and memory resources to meet the growing capability of data processing, the requirements of real-time control and advanced analysis computation.Smart cloud provide a variety of services in a transparent way to the user and power system application. It is a cloud computing system to use virtual computing and memory resource pool for dynamic deployment, dynamic allocation / distribution, real-time monitoring, thereby it could provide the requirements of Q\&S computing services, data storage service and platform services for the user or power system.

Use italic for emphasizing a word or phrase. Do not use boldface typing or capital letters except for section headings (cf. remarks on section headings, below).

\section{The Construction of Smart Cloud}

The computing environment of smart cloud can be divided into three basic levels: the physical resource layer, the platform layer and the application layer.The physical resource layer includes a 
variety of computing resources and storage resources which can also be provided the users as a service, that IaaS (infrastructure as a service). IaaS provides the users not only for virtualized computing and storage resources, but also to ensure that the users bandwidth when accessing the network.

The platform layer is the most critical layer of smart cloud computing and is the connection link of the upper applcation and lower resources.Its function is to shield the heterogeneous characteristics of a variety of distribution resources and manage its effective, in order to provide a consistent and transparent interface for the application layer.

The platform layer is the core of smart cloud computing system which including advanced application of smart grid, the design and development environment of real-time control program, the storage management system and file system of mass data, the other systems management tools which could realize the intelligent cloud computing. Such as the resource deployment, distribution, monitoring management, security management, and distributed concurrency control in the smart cloud computing system. The platform layer provides the application program running and maintenance needs of all platform resources for application developers, the developers do not have to worry about the needed resources when applying. The platform layer reflects that the platform is a service which known as PaaS ( platform as a service ).

The application layer is the embodiment of user demand. It applies in the smart gird through a variety of tools and the specific development environment. It is oriented to users with the software application services and user interaction interface, so this layer is called the software as a service, namely SaaS (software as a service).

\section{The Resource Management of Intelligent Cloud}

The resource loaded in the smart cloud computing environment is different at different time, and mass application and services data of smart grid will led to the growth of failure probability which could make resources are always change. In addition, the resource ownership is distributed, all levels grid have certain computing resources and storage resources, different resource provider could impose different constraints on their resources,all that leading to run a unified management strategy in the whole environment is difficult. Therefore, if using the centralized architecture,the smart only set one resource management system in the cloud environment.It is likely to cause bottlenecks and a single point of failure that could make some problems of the whole environment in stability, reliability and flexibility, it is not suitable for large-scale smart cloud computing environment.

The idea of solving above problem is to introduce the distributed resource management architecture with the domain model. This model divides the smart cloud computing environment into two levels: the first level is some logical units, we call it the management domain which is composed of some resources of the power grid, such as the high performance computing, massive database consisting of an autonomous system.Each administrative domain has its own local resource management system which is responsible for managing the various resources of the region; the second level is the whole smart cloud computing environment which constituting of the connected mutually management domain.

Management domain represents the maximum range of the centralized resource management and the basic unit of the distributed resource management that it reflects good fusion of the two mechanisms.In order to ensure the consistency of system behavior and management strategy in the domain,the local resource management system of each domain should centralized organize and manage its resource information. Multiple administrative domains could provide the global resources for the whole smart cloud computing environment through by mutual cooperation that it is convenient resource users to access. The internal structure of each domain is transparent for resource users.After introducing the management domain into the system, the composition of smart cloud is shown in the Figure 2. 


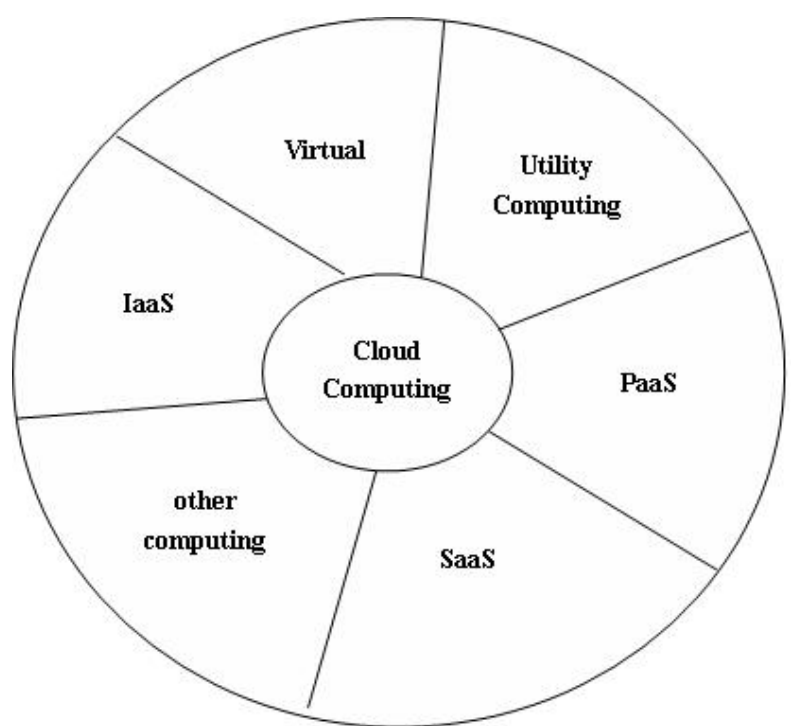

Fig. 2 Concept Formation of Cloud Computing

\section{The Security Strategy of Smart Cloud}

The resource of smart cloud computing environment is shared, at the same time there may be multiple users or applications need to request the providing services of these resources. As the creation objects of these applications are usually different,they also have different security requirements.It needs providing different security strategies for achieving these requirements.

The implement of traditional security strategies are required by the resource provider, it must notice all the associated resource whenever any resource provider changes, this would inevitably lead to high management cost and greatly increase the resource provider's burden. In addition, the safety assessment contents of the strategy is too much that it is difficult to prevent security breaches and eventually it will lead to its safety of smart cloud resources sharing can' t be guaranteed. In order to flexibly manage each member and decide who is the developer, who is the user, and use all kind of services of the provided resources respectively, and allow to adjust the members and own their rights in any time. All that needs the ability of smart cloud within the formulation and implementation of fine-grained security policy ( especially the access control policy ).

Due to the introduction of the resource management domain, the smart cloud computing environment should be composed of multiple resource management domains.Correspondingly, the security policy of smart cloud computing is divided into internal domain security policy and global security strategy.Internal domain security policy only manages the internal domain, while the global security strategy refers to various interactive safety strategy between the impactor of smart cloud and management domain.

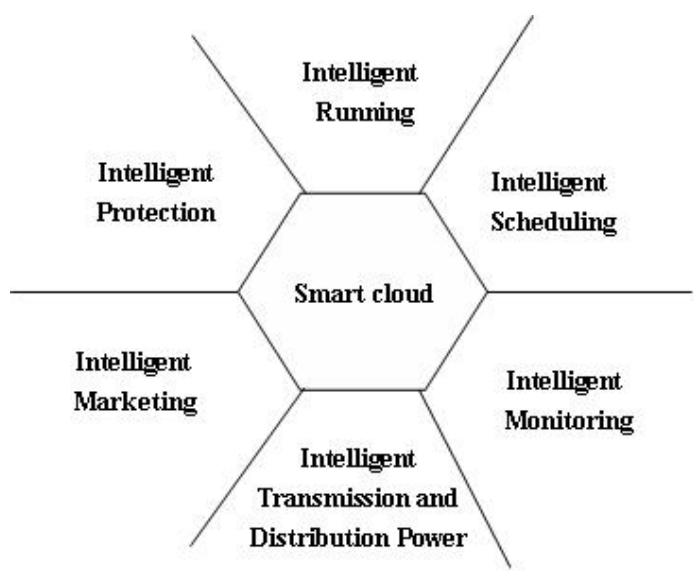

Fig. 3 Component Link of Smart Cloud

Internal domain security policy is defined as a group of rules that all the related parameters of the management domain and internal domain security.It launches a variety of security related activities in the domain, such as the use of the user name,password login or using SSH to implement remote operation,all that is the specific embodiment of the safety strategy.Global security strategy is the 
various interactive behavior between smart cloud and $\mathrm{m}$ the external management domain which decide to how to access the provided services by the management domain resource.Fox example, what are the characteristics of ( including identity, attribute) member can access which service resources of the domain. It is responsible for the internal domain policy evaluation and harmonic of smart cloud computing environment, together acting on various interactive behavior in the smart cloud computing environment, by this way can constitute a complete security strategies.

\section{Summary}

Cloud computing is the further development of the distributed computing, parallel processing and grid computing which is based on the Internet computing.It is a system that can able to provide hardware services, infrastructure services, service platform, software services and storage services for a variety of network applications. Smart grid represents the future development trend of electric power industry which is the fusion of advanced network communication technology, information processing technology and modern network technology. Therefore, cloud computing technology is introduced into smart grid field that fully exploit the computation capability of the existing power system and storage facilities, to enhance its applicability and utilization.Doubtlessly,it has very important research value and significance.

\section{Acknowledgments}

This work was supported by the Key Project of Henan Province (132102210385;142102210042); the

Science and Technology Key Project for the Education Department of Henan Province(13B413968,14B120010); The Scientific and Technological Project of Zhengzhou City (121PPTGG418)

\section{References}

[1] Pan Rui, Liu Junyong, Guo Xiaoming.Study Power System on Cloud Computing[J].Sichuan Electric Power Technology,2010,33 (3): 71-76

[2] Li Qilin, Zhou Mingtian, Smart Grid and Its Key Technology Research[J].Computer Science,2010,37(8):102-104

[3] Chen Quan, Deng Qianni.Cloud Computing and Its Key Technology[J]. Computer Application,2009,29( 9):2562-2567

[4 ] Dikaiakos M.D., Katsaros D, Mehra.Cloud Computing: Distributed Intemet Computing for IT and Scientific Research[J].Intemet Computing, IEEE,2009,13(5): 10-13

[5] Zhang Jianxun, Gu Zhimin, Zheng Chao, cloud computing, research progress[J]. application research of computers, 2010,27(2):429-433

[6] Zhang Wenliang, Liu Zhuangzhi, Wang Mingjun, Yang Xusheng. Smart Grid Research and Development Trend[J].Grid Technology,2009, 33(13): pp.1-11 